\title{
A PCB-Based Electronic ELISA System For Rapid, Portable Infectious Disease Diagnosis
}

\author{
Konstantinos I. Papadimitriou, Daniel Evans, Hywel Morgan and Themistoklis Prodromakis \\ Nanoelectronics \& Nanotechnology Research Group, School of Electronics and Computer Science \\ University of Southampton, Southampton, SO17 1BJ, UK \\ Email: $\{$ kp1y13, dje1r15, hm, tp1f12\}@ecs.soton.ac.uk
}

\begin{abstract}
In this paper an amperometric electrochemical detection method is demonstrated and implemented using exclusively Printed Circuit Board (PCB)-based technologies. A portable, reconfigurable, multichannel amperometric dataacquisition board has been designed and fabricated, dedicated to the measurement of current-input signals delivered by the PCB-based biosensor. The electronic read-out circuit is able to provide constant biasing voltages to the amperometric sensor, measure in real-time the sensor's output currents, digitise them using high-accuracy Analog-to-Digital Converters (ADCs) and send the binary data to the user either through a USB2.0 interface or via an on-board TFT touch-screen. In order to validate the robustness and accuracy of the combined system, proof-ofconcept amperometric experiments have taken place using our custom-made PCB-based system and standard electrochemical substrates. The results obtained have been cross-validated by means of standard colorimetric analysis and their differences have been highlighted and analysed.
\end{abstract}

Keywords-Amperometry, Analog-to-Digital Conversion, Data Acquisition, Electrochemical Assay, ELISA, PCB-Based Sensor, Point-Of-Care Diagnostics, TMB

\section{INTRODUCTION}

The modern era has seen extraordinary advances in medical science and analytical technologies and our understanding of the precise biomolecular epidemiology that underlies pathological processes has improved considerably [1]. Diagnostic and prognostic biomarkers for many common diseases are being revealed in much greater detail, driven by significant advances in detection technology and sensitivity. As a result, diagnostic systems are required to identify biomarkers at very low concentrations, e.g. pg/mL. Furthermore, increased biomolecular analysis as part of the diagnostic process requires a higher level of diagnostic efficiency and portability. For these reasons traditional "gold standard" techniques such as the Enzyme Linked Immuno-Sorbent Assay (ELISA) are evolving towards full automation and more efficient methodologies of signal resolution. Amperometry has been shown to be an effective conduit for ELISA signal detection due to the electrochemically active nature of many existing colorimetric and fluorimetric reporter compounds [2], [3].

We present the natural progression of amperometric research technology into a lightweight, versatile, low-cost assay system manufactured fully on PCBs. Assay technology has been engineered to occur directly at the PCB surface and amperometric detection of a common colorimetric product is demonstrated at higher sensitivity than is allowed by colorimetric protocols. Thus, the system can be used to significantly increase the sensitivity of most industry standard diagnostic ELISA kits and improve their portability, due to the exclusion of bulky high-sensitivity spectrometric apparatus. With this technology we can quantify biochemical reporters using standard electronic techniques, both in analog and in digital domain, without the need to convert the optical signal into a current or voltage.

In Point-of-Care (POC) applications, a handheld, electronic ELISA ("eELISA") has long been viewed as the next step in future diagnostic technology. In order to provide accurate electrical colorimetrically analogous results from an "eELISA", an electronic read-out circuit is required. In this paper, we propose a complete, proof-of-concept system, including a multi-channel data acquisition board and a passive PCB-based sensor, where the molecular "eELISA" is taking place. The preliminary results obtained with the described technology, allow us to proceed with an embedded system, where the readout electronics and biosensors would communicate in a fullyintegrated user-friendly manner within an architecture that permits multiplexed detection across a range of interchangeable assay chips [4], [5].

We discuss initially the concept of the "eELISA" and present the data-acquisition board in detail. Subsequently, we show preliminary amperometric analyses with comparison to existing colorimetric systems. Finally, we offer some conclusions and future prospects for the presented technology.

\section{The "eELISA" Detection APPROACH}

The amperometric detection method utilised in this analysis is often described as second generation amperometry [6]. Unlike other amperometric approaches this system does not rely on reactions occurring at the electrode but measures charge carrier concentration directly in the form of distinct current levels. In order to integrate amperometric detection into standard immunodiagnostic assay systems it is necessary only that the reporter component deliver a measurable change in charge carriage capable species.

A reporter system commonly employed in colorimetric ELISAs is the enzymatic conversion of $3,3^{\prime}, 5,5^{\prime}$ Tetramethylbenzidine (TMB) to the corresponding diimine by the horseradish peroxidase (HRP) enzyme (TMB is colourless, while the diimine product is bright blue) [7]. Hydrogen peroxide $\left(\mathrm{H}_{2} \mathrm{O}_{2}\right)$ is consumed as a co-factor in this process, providing free hydroxyl ions to the solution. Both the TMB diimine product and hydroxyl ions are charge carriage capable and therefore electrochemically detectable, thus, theoretically, 


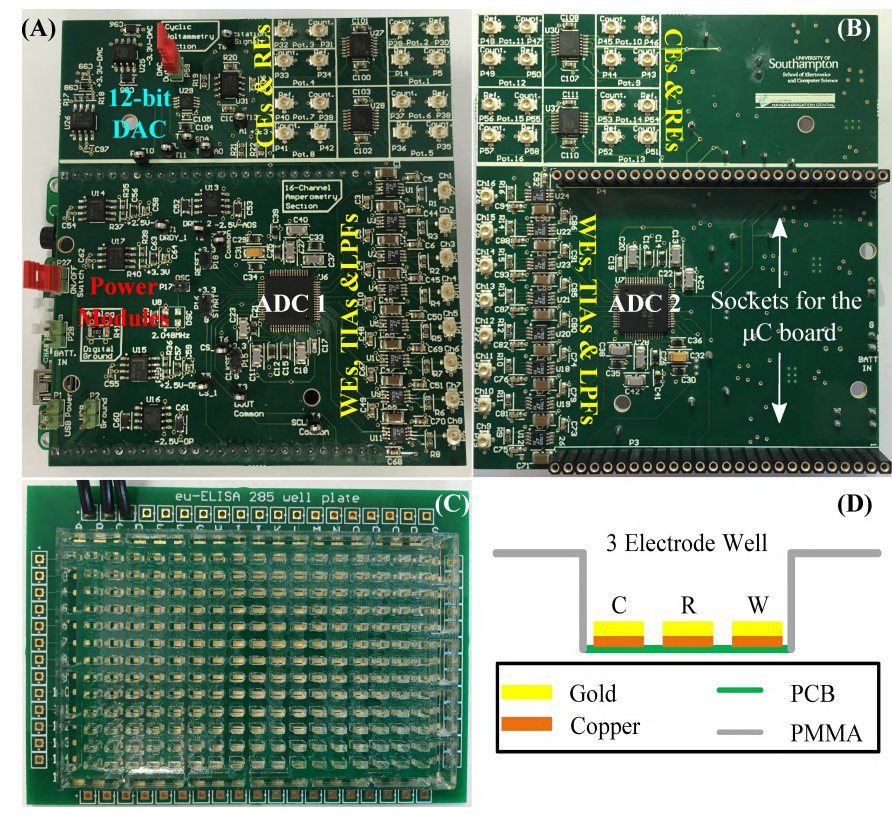

Fig. 1. The proposed "eELISA" system. In (A) and (B) the top and bottom side of the boinstrumentation board can be seen, respectively. On the top layer the ADC for the first eight channels is located, while on the bottom layer the second ADC for the remaining eight channels can be seen. In (C) the PCBbased sensor is shown with its 285 sensor plates, while in (D) a schematic representation of a cross-section of the sensor's architecture is provided.

allowing any standard TMB assay to be assessed amperometrically. Our intention includes the improved sensitivity of existing immunodiagnostic assay systems through the replacement of standard colorimetry with amperometric detection.

\section{A. The PCB-Based Amperometric Sensor}

The fabricated PCB-based sensing system can be seen in Figure 1(C). The sensor surface is comprised of three gold electrodes on a PCB forming working, counter and reference electrodes. Gold provides a stable amperometric sensor surface and allows thiol linkage to single chain cysteine modified antibody fragment $\left(\mathrm{Fab}^{\prime}\right)$ so that diagnostic assay chemistry can be performed directly at the sensor. All sensor surfaces measure $2.4 \times 1.2 \mathrm{~mm}$ and are commercially fabricated by deposition of a thin layer of gold over a standard thickness copper contact pad. Wells were fabricated from PDMS and fixed to the PCB to allow introduction of samples to the electrode surfaces under conditions through which the current path in the electrolyte could be controlled. A schematic representation of a crosssection of the sensor's architecture is shown in Figure 1(D).

\section{B. The Multichannel Amperometric Bioinstrumentation Board}

An electronic multi-channel, reconfigurable, portable bioinstrumentation platform has been designed, fabricated and programmed for the appropriate interface with the aforementioned PCB-based sensors. A view of the top and bottom layers of the fabricated board can be found in Figure 1(A) and (B), where all its distinct compartments are marked-up. A commercially available development board which includes an ATxmega128A1 microcontroller for the control of the various Integrated Circuits (ICs) and a 2.8-inch TFT touch-screen has been connected to the bottom layer of our custom-made PCB using standard board-to-board connectors.

Two, fully-differential eight channel, 16-bit resolution voltage-input ADCs have been employed for the digitisation of the converted current values received from the front-end low-noise transimpedance amplifiers (TIAs) LTC2055. All 16 TIAs include $0.1 \%$ tolerance resistors for precise and matched current readings and appropriate value capacitors for the filtering of high-frequency noise. The ADCs operate with bipolar power supply $( \pm 2.5 \mathrm{~V})$ and thus, are able to detect positive and negative reaction currents. The overall design of the board revolves around the idea of immediate digitisation of the analog biosensor signals, process them by means of standard DSP techniques and finally send them to the user in digital or in analog form [8]. The system can be powered-up via a USB-connection or by a single $5 \mathrm{~V}$ battery, ensuring its portability. A simplified schematic diagram of the complete electronic platform is shown in Figure 2, including the data retrieval timings for the Serial Peripheral Interface (SPI) communication protocol been used for the two ADCs.

With the use of the 12-bit, single-output Digital-to-Analog Converter (DAC) AD5321 and an appropriate circuitry, the bioinstrumentation board can provide bipolar biasing voltages to the sensor from $-3.3 \mathrm{~V}$ up to $+3.3 \mathrm{~V}$ with a resolution of $\sim 1.6 \mathrm{mV}$, using the standard three-electrode potentiostat architecture (see Figure 2). The electronic system is able to read-out simultaneously from 16-channels in a real-time manner and dynamically change the gain of the internal Programmable Gain Amplifiers (PGAs) of the ADCs, if the readings are below certain threshold values. Moreover, through the onboard DAC, the user is able to perform a Cyclic Voltammetry (CV) calibration before every measurement, if necessary. Table I summarises the characteristics of the fabricated board.

TABLE I. BIOINSTRUMENTATION BOARD's CHARACTERISTICS

\begin{tabular}{lc}
\hline Input current range $(\mathrm{dB})$ & 90.3 \\
Input current resolution (nA) & 122 \\
ADCs resolution (bit) & 16 \\
ADCs data rate (kSPS) & $0.125-8$ \\
\hline Board dimensions (mm) & $83 \times 93$ \\
DSP unit & ATxmega128A1 - 32MHz \\
Total current required for & $\sim 45$ \\
the fabricated PCB of Figure 1 (mA) & $5 \mathrm{~V}$ (Battery or USB) \\
Power supply & \\
\hline
\end{tabular}

\section{EleCtRochemicAl ReSUlts}

We require a system that shows clear definition between measured sample currents but we also wish to minimise the biasing voltage to reduce damage observed at the gold sensor surface following repeated measurements. Performance was assessed through standard CV under different biasing voltages to establish effective sensor calibration. Negative bias values from 0 to $-400 \mathrm{mV}$ with $50 \mathrm{mV}$ steps were investigated. Analytes of various TMB product concentration were produced as described below and three distinct samples corresponding to low, medium and high colorimetric levels were measured at each biasing voltage. $\mathrm{CV}$ showed $-300 \mathrm{mV}$ to provide maximum signal elevation and thus, optimum system sensitivity, without showing excessive deterioration of sensor surface integrity under extended or repeated analysis.

Subsequently, a number of experiments were conducted to verify the performance of our system. The sensor's drift was 


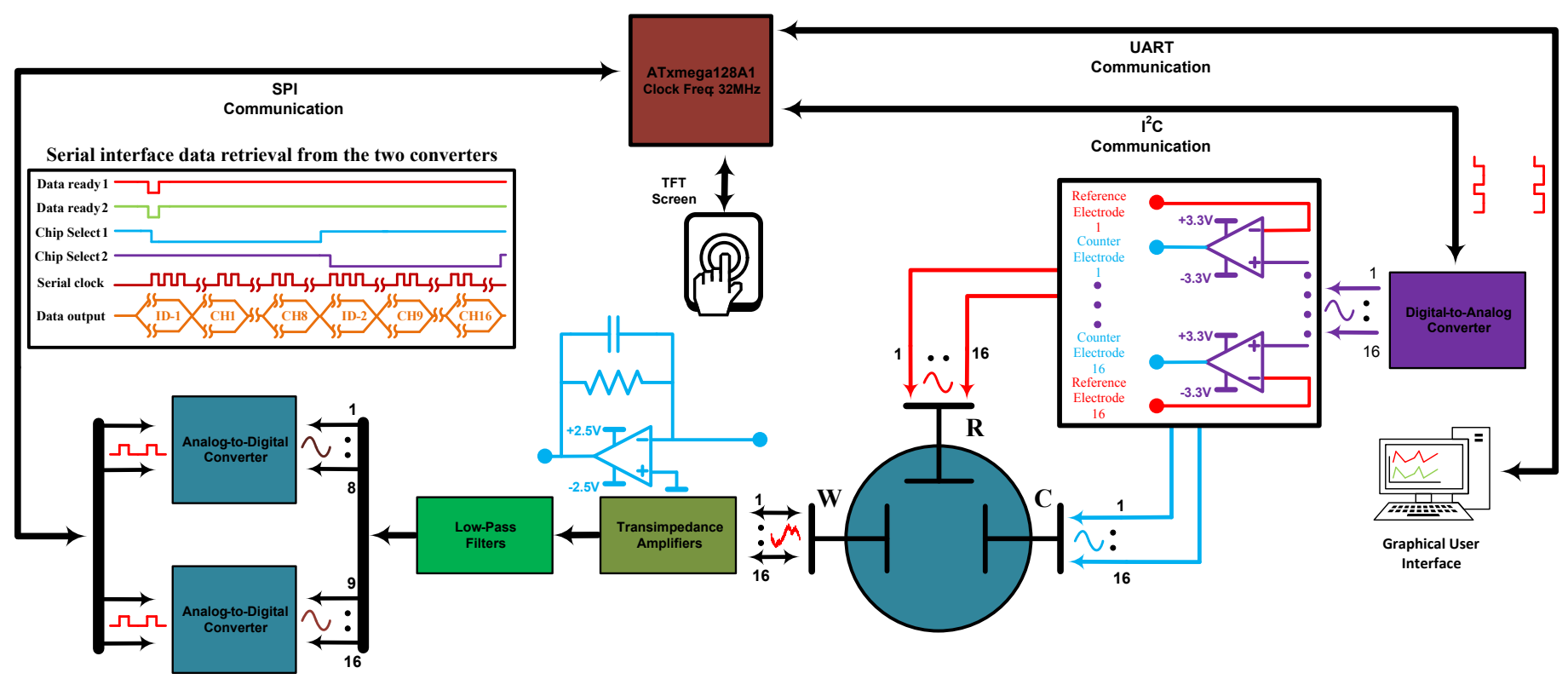

Fig. 2. A simplified schematic diagram of the fabricated bioinstrumentation board that interfaces with the PCB-based sensor.

assessed by measuring the current delivered across three TMB product solutions at high, medium and low concentrations (relative to the full colorimetrically detectable range). A $5 \mathrm{mN}$ $\left(\mathrm{PO}_{4}^{3-}\right)$ phosphate-buffered saline (PBS) was included to highlight drift occurring due to electro-adsorption of components of the HRP-TMB reporter systems to the sensor surface. The aforementioned $-300 \mathrm{mV}$ constant bias was applied to the reference electrode and current recorded over three minutes (a x6 time period, since the proposed assay time is $30 \mathrm{~s}$ ). The results are shown in Table II.

TABLE II. PCB-BASED SENSOR DRIFT

\begin{tabular}{ccccc}
\hline & High $[\mathbf{T M B}]$ & Medium $[\mathbf{T M B}]$ & Low $[\mathbf{T M B}]$ & PBS $(\mathbf{5 m N})$ \\
\cline { 2 - 5 } Drift $(\mathrm{nA} / \mathrm{s})$ & 180 & 112 & 86 & 13.3 \\
\hline
\end{tabular}

The observed linearity demonstrates quantification capable measurement during the time window of interest and that our chosen analysis time of 30 seconds is appropriate. Comparison of TMB samples (containing biological components such as HRP) to PBS alone shows that some of the drift is attributable to sample composition and is suggested to result from electrochemical adsorption to the sensor surface, higher concentrations of biological components lead to higher levels of drift. We suggest that non-biologically related sensor drift results from progressive sensor surface degradation.

Next we assessed TMB product detection in comparison to the colorimetric system. Analytical samples containing roughly titrated quantities of detectable TMB product were achieved by preparing a 12 point $\log _{2}$ dilution scheme of HRP in deionised $\mathrm{H}_{2} \mathrm{O}$ and adding $10 \mu L$ of each dilution to $100 \mu \mathrm{L}$ of freshly prepared TMB reagent (1 TMB tablet -Sigma T5525- dissolved in $10 \mathrm{~mL} 0.014 \% \mathrm{H}_{2} \mathrm{O}_{2}$, centrifuged $16850 \mathrm{RCF} / 1 \mathrm{~min}$ to remove particulates and decanted to a fresh tube). When appropriate colorimetric values had been achieved the reaction was stopped by addition of $10 \mu L 1 \mathrm{M}$ $\mathrm{H}_{2} \mathrm{SO}_{4}$. Samples were then measured colorimetrically using a Promega Glomax ${ }^{\circledR}$ plate reader, and amperometrically using the bioinstrumentation board described herein. Amperometric sensor surfaces were rinsed $\mathrm{x} 1$ with $2 \mathrm{M} \mathrm{HCl}$ and $\mathrm{x} 3$ with $\mathrm{H}_{2} \mathrm{O}$ between measurements. The produced current was measured for 30 seconds and final endpoint values collected. The resulting data in Figure 3 demonstrate that the amperometric linear detection range of TMB product concentration extends throughout and beyond that of colorimetric detection allowing a greater range of sample concentrations to be assessed through a single reagent protocol. The correlation is closely linear as required for effective biodetection.

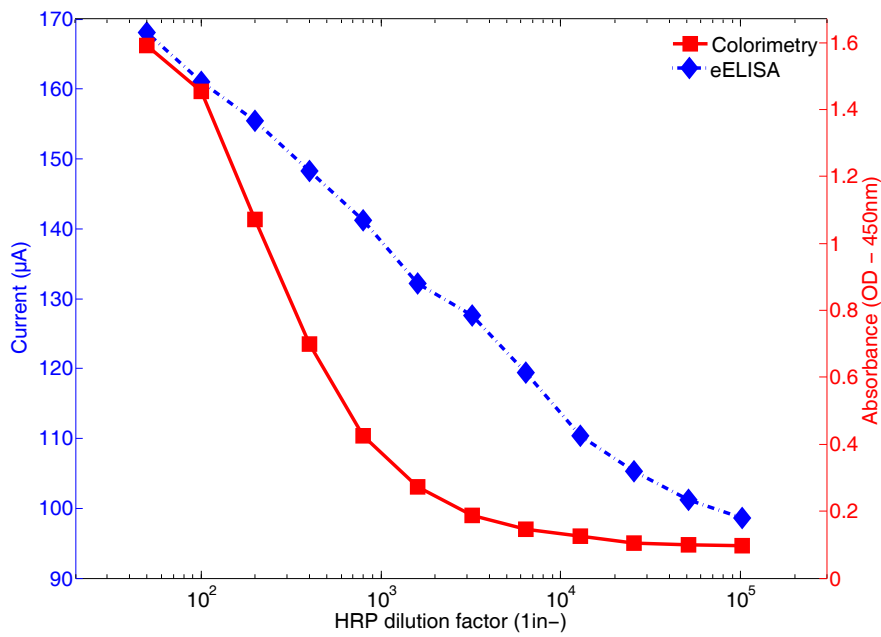

Fig. 3. Comparative colorimetric versus amperometric sensing results for a $\log _{2}$ dilution scheme of HRP.

Further investigation was conducted by preparing a shallower HRP dilution scheme $\left(\log _{1.25}\right)$ to analyse amperometric detection resolution at lower TMB product concentrations and the results are presented in Figure 4. We demonstrate that the linear correlation between TMB product and amperometric response extends to concentrations that are essentially unde- 
tectable by colorimetry. This discovery implies that the system will naturally allow the higher assay sensitivity required in modern medical biomarker analyses. In many colorimetric ELISAs, assay sensitivity is limited by the resolution in optical detection of the assay product. Here, we have shown that TMB product concentrations that are undetectable by optical density can be discriminated by amperometry thus, allowing improved assay sensitivity without changing any molecular aspects of the assay or protocol.

We further analysed our low concentration detection results of Figure 4 by expressing the difference between sequential points as a percentage increase and plotting the results in Figure 5. A broadly linear increase is seen for the whole amperometric dataset, while the colorimetric data indicate that progressive absorbance in the low concentration analysis only occurs at the highest two points. Thus, highlighting the improved detection sensitivity provided by the described amperometric system.

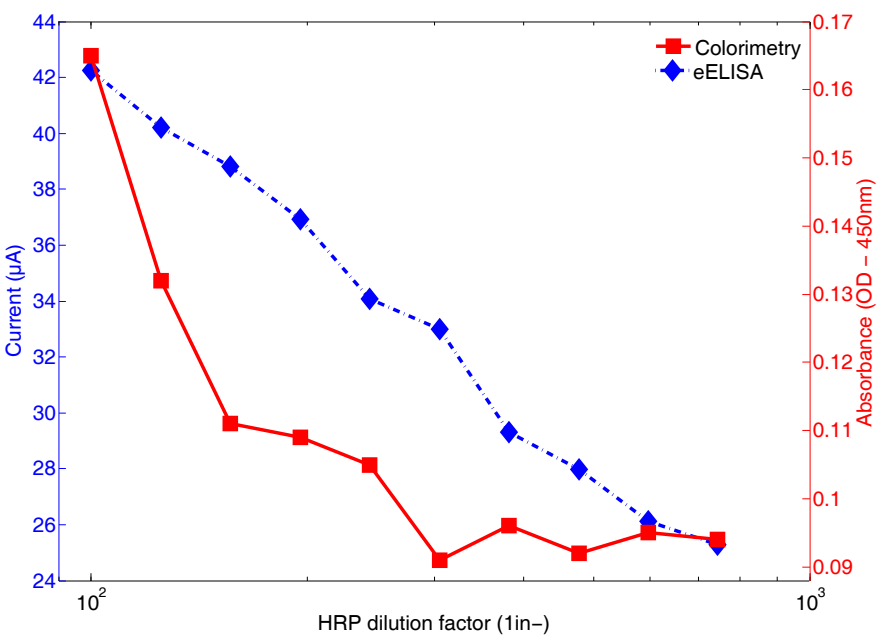

Fig. 4. Colorimetric versus amperometric sensing results for a $\log _{1.25}$ dilution scheme of HRP allowing measurement of lower TMB product concentrations, compared to Figure 3. (The final TMB product concentration depends on both HRP dilution and development time, thus, cross-comparison of Figure 3 and Figure 4 is achieved by colorimetric values rather than HRP dilution factor.)

\section{CONCLUSiON}

This paper presents an electrochemical detection platform, developed using commercially available PCB technologies. The proposed system comprises the custom-made sensor and a multi-channel bioinstrumentation board, built entirely from discrete "off-the-shelf" components. The electronic board can be powered-up by a battery or via USB connection, depending whether a lab- or POC-based application is required. The presented experimental results demonstrate that the behaviour of the "eELISA" approach is similar to the standard colorimetric ELISA protocol and more specifically, in lower concentrations the "eELISA" exhibits higher sensitivity than the colorimetric method. These encouraging results allow us to proceed to the development of a final POC system version, which will combine a similar electronic board with a PCBbased sensing platform, including an embedded PCB-based microfluidic network [9].

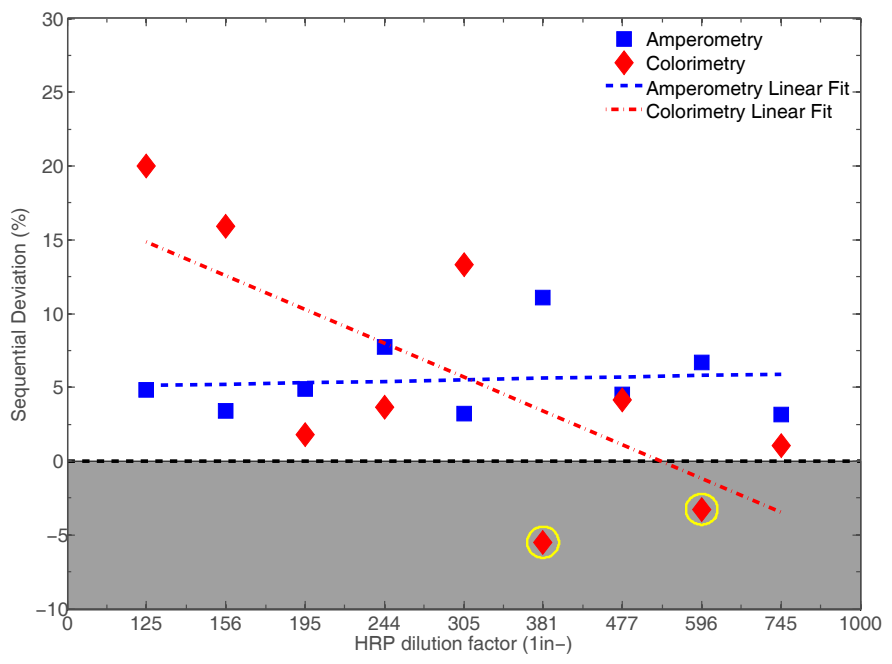

Fig. 5. Percentage difference within the obtained amperometric and colorimetric results. The "eELISA" method demonstrates a roughly linear signal increase in response to TMB product concentration at levels producing no consistent colorimetric readings.

\section{ACKNOWLEDGMENT}

The authors acknowledge the financial support of the Engineering and Physical Sciences Research Council (EPSRC), EP/L020920/1 research grant. The authors also thank their industrial partner Newbury Electronics Ltd. for the fabrication of the custom-made PCB-based biosensors.

\section{REFERENCES}

[1] R. McNerney and P. Daley, "Towards a point-of-care test for active tuberculosis: obstacles and opportunities," Nature Reviews Microbiology, vol. 9, no. 3, pp. 204-213, 2011.

[2] A. Bhimji, A. A. Zaragoza, L. S. Live, and S. O. Kelley, "Electrochemical enzyme-linked immunosorbent assay featuring proximal reagent generation: Detection of human immunodeficiency virus antibodies in clinical samples," Analytical chemistry, vol. 85, no. 14, pp. 6813-6819, 2013.

[3] C. Hu, I. Zeimpekis, K. Sun, S. Anderson, P. Ashburn, and H. Morgan, "Low-cost nanoribbon sensors for protein analysis in human serum using a miniature bead-based enzyme-linked immunosorbent assay," Analytical chemistry, vol. 88, no. 9, pp. 4872-4878, 2016.

[4] T. Prodromakis, Y. Liu, J. Yang, D. Hollinghurst, and C. Toumazou, "A novel design approach for developing chemical sensing platforms using inexpensive technologies," in Biomedical Circuits and Systems Conference (BioCAS), 2011 IEEE, Nov 2011, pp. 369-372.

[5] T. Prodromakis, Y. Liu, and C. Toumazou, "A low-cost disposable chemical sensing platform based on discrete components," Electron Device Letters, IEEE, vol. 32, no. 3, pp. 417-419, 2011.

[6] C. Chen, Q. Xie, D. Yang, H. Xiao, Y. Fu, Y. Tan, and S. Yao, "Recent advances in electrochemical glucose biosensors: a review," Rsc Advances, vol. 3, no. 14, pp. 4473-4491, 2013.

[7] P. Fanjul-Bolado, M. B. González-García, and A. Costa-García, "Amperometric detection in tmb/hrp-based assays," Analytical and Bioanalytical chemistry, vol. 382, no. 2, pp. 297-302, 2005.

[8] K. I. Papadimitriou, C. Wang, M. L. Rogers, S. A. Gowers, C. L. Leong, M. G. Boutelle, and E. M. Drakakis, "High-performance bioinstrumentation for real-time neuroelectrochemical traumatic brain injury monitoring," Frontiers in Human Neuroscience, vol. 10, p. 212, 2016.

[9] N. Vasilakis, D. Moschou, D. Carta, H. Morgan, and T. Prodromakis, "Long-lasting fr-4 surface hydrophilisation towards commercial pcb passive microfluidics," Applied Surface Science, 2015. 